\title{
Practical Universal Method for Designing Single-Layer Electromagnetic Wave Absorbers
}

\author{
Benjamin Jacard*, Alvaro Valenzuela, Manuel Gonzalez \\ Department of Electrical Engineering, University of Chile, Santiago, Chile \\ Email: *bjacard@ing.uchile.cl
}

How to cite this paper: Jacard, B., Valenzuela, A. and Gonzalez, M. (2018) Practical Universal Method for Designing Single-Layer Electromagnetic Wave Absorbers. Open Journal of Antennas and Propagation, 6, 84-92.

https://doi.org/10.4236/ojapr.2018.64008

Received: October 6, 2018

Accepted: December 17, 2018

Published: December 20, 2018

Copyright $\odot 2018$ by authors and Scientific Research Publishing Inc. This work is licensed under the Creative Commons Attribution International License (CC BY 4.0).

http://creativecommons.org/licenses/by/4.0/

\begin{abstract}
A simple design method for electromagnetic wave absorbers under normal incidence is presented. For the fabrication of the microwave absorbing material a novel design chart is used, including curves of constant $\delta_{e} / \delta_{m}$ ratio in a $\left(\delta_{e}+\delta_{m}, \varepsilon^{\prime} / \mu^{\prime}\right)$ plane. The practical use of the chart is explained and, as example, a microwave absorber fabricated with magnetite-impregnated plastisol is designed and tested at X-band frequencies.
\end{abstract}

\section{Keywords}

Permittivity, Permeability, Single Layer, Microwave Absorption, Reflection Loss

\section{Introduction}

The single-layer homogeneous absorber (SLHA) backed by a perfectly conducting plate, known also as the Dallenbach layer, has been the subject of several investigations in the last decades due to its practical importance [1]-[9]. According to Figure 1, the design parameters for the SLHA are: $\varepsilon^{\prime}, \varepsilon^{\prime \prime}, \mu^{\prime}, \mu^{\prime \prime}$ and $d / \lambda_{0}$. These parameters must satisfy the zero reflection condition which occurs when the surface impedance of the structure becomes identical to that of free space. In order to simplify the design process, graphical aids have been presented providing also a view of the interrelated numerical values of material parameters required to meet the perfect matching condition at the design frequency [1]-[9].

All design theories for SLHA compute the reflection coefficient at the interface, usually in terms of the input impedance at the absorbing layer. The general solution for the perfect SLHA was found by defining the electric loss angle $\delta_{e}=\arctan \left(\varepsilon^{\prime \prime} / \varepsilon^{\prime}\right)$ and the magnetic loss angle $\delta_{m}=\arctan \left(\mu^{\prime \prime} / \mu^{\prime}\right)$ as independent variables to present the zero reflection condition in the $\left(\delta_{e}, \delta_{m}\right)$ plane 


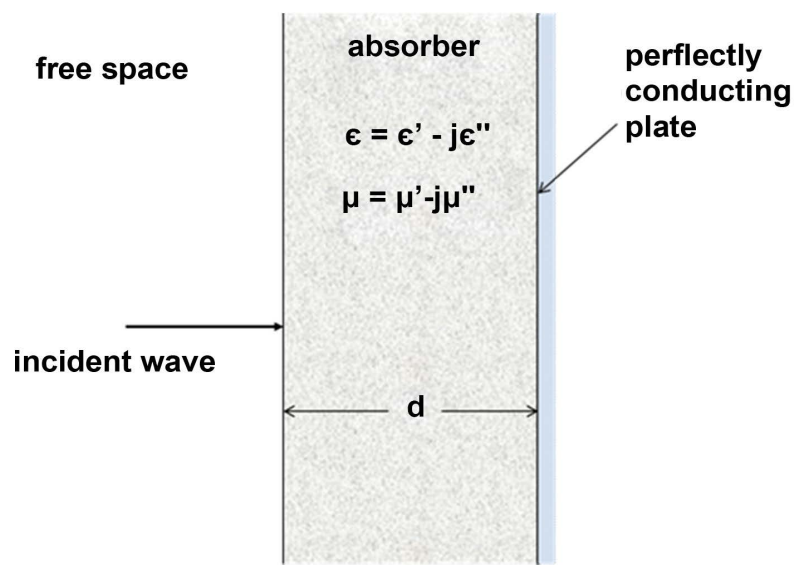

Figure 1. Homogeneous layer absorber.

[3]. Within this original approach, the first design condition that the input impedance is real, fixes the electric thickness of the layer. Finally, the condition that the input impedance is equal to the intrinsic impedance of free space yields the $\mu^{\prime} / \varepsilon^{\prime}$ ratio required for perfect absorption. This general solution was further developed and extended to calculate previously unavailable universal design curves and design equations for different orders of solutions [8].

Using the same theoretical approach employed to obtain general solutions [3] [8], in this paper we present a novel universal design chart for the SLHA where instead of curves of constant $\mu^{\prime} / \varepsilon^{\prime}$ ratio in the loss plane $\left(\delta_{m}, \delta_{e}\right)$, curves of constant $\delta_{e} / \delta_{m}$ ratio in the $\left(\delta_{e}+\delta_{m}, \varepsilon^{\prime} / \mu^{\prime}\right)$ plane are presented.

Absorbers are usually fabricated by impregnating a dielectric binder with a lossy filler; the main contribution of this work is to facilitate the design of the SLHA, by providing a graphical aid that shows when the optimum filler to binder ratio that gives perfect absorption has been achieved. Another contribution is the successful use and measurements of natural magnetite-impregnated plastisol as microwave absorbing material since the electromagnetic characteristics and the use of this composite at X-band frequencies seemingly have not been reported in the literature.

This paper is organized as follows: Section 2 provides the theoretical approach for developing a universal design chart for the SLHA. Section 3 explains the practical use of the chart for designing a microwave absorber. As a practical demonstration of this novel design procedure, an absorber fabricated with a composite material made of magnetite-impregnated plastisol is designed and tested at $\mathrm{X}$-band frequencies; the main results are presented and discussed. Section 4 summarizes the main conclusions of this work.

\section{Theory}

The material of the SLHA is characterized by a thickness $d$ and complex relative electromagnetic parameters $\varepsilon=\varepsilon^{\prime}-j \varepsilon^{\prime \prime}=\varepsilon^{\prime}\left(1-j \tan \delta_{e}\right)$ and $\mu=\mu^{\prime}-j \mu^{\prime \prime}=\mu^{\prime}\left(1-j \tan \delta_{m}\right)$. When imposing the zero reflection condition, the following equations are obtained [3] [8]: 


$$
\begin{gathered}
\frac{\varepsilon^{\prime}}{\mu^{\prime}}=\frac{\cos \delta_{e}(\cosh \theta s-\cos \theta)}{\cos \delta_{m}(\cosh \theta s+\cos \theta)} \\
\sin \theta=r \sinh \theta s
\end{gathered}
$$

where $\theta=4 \pi d / \lambda$ is the two-way electric length of the absorber, $\lambda$ is the wavelength inside the material, $r=\tan 1 / 2\left(\delta_{m}-\delta_{e}\right), s=\tan 1 / 2\left(\delta_{m}+\delta_{e}\right)$.

We depart from the charts presented in previous works [3] [8] and, by using the same theoretical framework, instead of providing curves of constant $\mu^{\prime} / \varepsilon^{\prime}$ ratio in the $\left(\delta_{m}, \delta_{e}\right)$ plane, we generate universal design curves of constant $\delta_{e} / \delta_{m}$ ratio in the $\left(\delta_{e}+\delta_{m}, \varepsilon^{\prime} / \mu^{\prime}\right)$ plane. The main advantage of this new approach is that in the later plane the electromagnetic properties of any type of material (binder and filler composite) can be plotted as a single point, whereas the former plane is suitable only for representing ideal absorbing conditions.

Using Equations (1) and (2), the universal design chart of Figure 2 has been produced. The chart contains curves for the two extreme cases of zero electric loss $\left(\delta_{e} / \delta_{m}=0\right)$ and zero magnetic loss $\left(\delta_{e} / \delta_{m}=\infty\right)$. No solutions can be found outside the band defined by the two extreme cases, the range of total loss $\left(\delta_{e}+\right.$ $\delta_{m}$ ) for perfect absorption is relatively narrow, especially for high $\varepsilon^{\prime} / \mu^{\prime}$ ratio. The intermediate condition of equal electric and magnetic loss angles $\left(\delta_{e} / \delta_{m}=1\right)$ is also presented for reference purposes.

These design curves are exact provided that the conditions of Figure 1 prevail, i.e. no gap between the absorber and the perfectly conducting plate. A particular design curve could be drawn for any intermediate value of $\delta_{e} J \delta_{m}$. However, this is not necessary when $\varepsilon^{\prime} \gg \mu^{\prime}$ since all solutions $\left(\delta_{e}+\delta_{m}\right)$ for total absorption will lie in a narrow band.

Microwave absorbing materials are usually prepared by blending a lossy filler in powder (e.g. carbon black, aluminum, iron, magnetite, ferrites) with a dielectric binder (e.g. epoxy resin, silicon rubber, plastics). These materials are characterized by a complex permittivity and permeability. When increasing the filler/dielectric ratio (maintaining constant frequency), the $\varepsilon^{\prime}, \delta_{e}$ and $\delta_{m}$ values increase [10] [11] [12] [13] [14]. Therefore, the corresponding points for the candidate absorber composites in the $\left(\delta_{e}+\delta_{m}, \varepsilon^{\prime} / \mu^{\prime}\right)$ plane of Figure 2 will outline a curve with positive slope that intersects the appropriate design curve on the chart, thus helping to determine the optimum filler concentration for the absorbing material. The design of the SLHA is completed specifying the material thickness $d$. For this purpose the following approximate solution of Equation (2) can be obtained [3] [8]:

$$
\frac{d}{\lambda_{0}}=\frac{\theta}{4 \pi} \frac{\lambda}{\lambda_{0}}=\left[\frac{\pi-r(\sinh \pi s-\pi s \cosh \pi s)}{1+r s \cosh \pi s}\right] \frac{1}{4 \pi} \frac{\sqrt{\cos \delta_{m} \cos \delta_{m} \cos \delta_{e}}}{\sqrt{\mu^{\prime} \varepsilon^{\prime}} \cos \frac{1}{2}\left(\delta_{m}+\delta_{e}\right)}
$$

This approximation has an error smaller than $1 \%$ for $\delta_{\mathrm{e}} \leq 0.6$ and $\delta_{m} \leq 0.7$ [8]. This loss range covers the design of most practical SLHA, outside this range, Equation (3) can be used as a first approximation to solve transcendental Equation (2). 


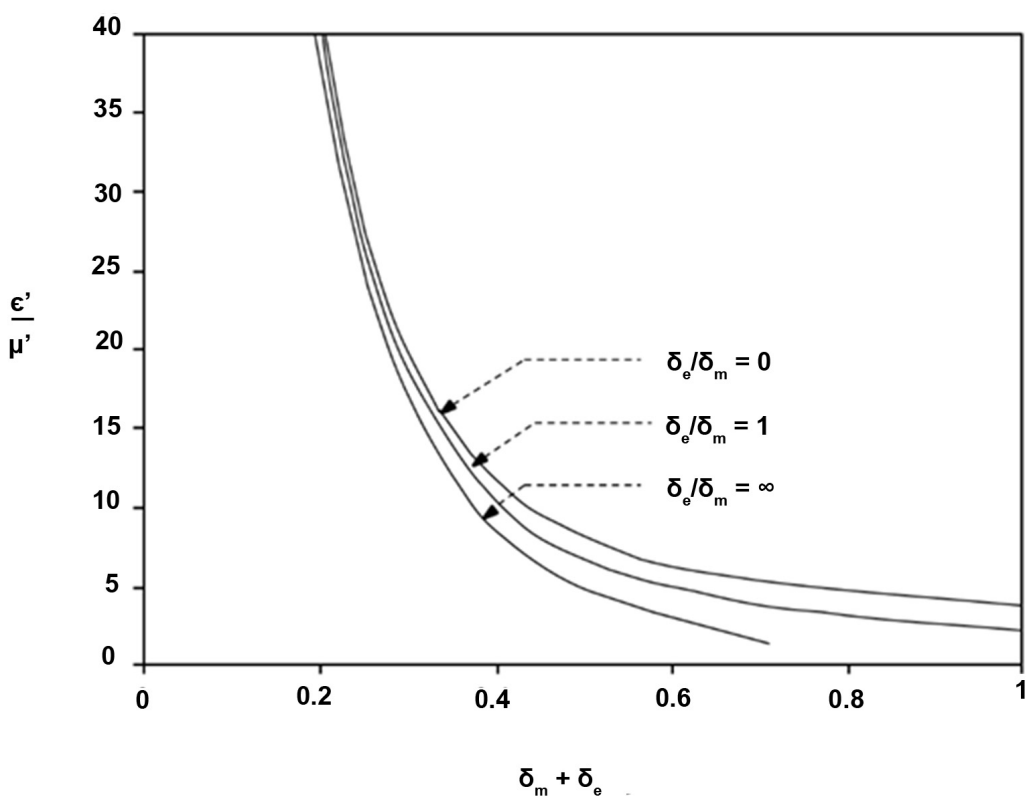

Figure 2. Universal design chart for SLHA.

When the loss angles are small, Equation (3) simplifies to the well-known quarter wavelength approximation $d=\lambda / 4$.

\section{Results and Discussion}

The design method will be illustrated using a composite made of magnetite-impregnated plastisol. The binder is a PVC emulsion and plasticizer compound. The filler is natural magnetite in powder sieved under 325 mesh (44 $\mu \mathrm{m})$. The components are blended according to a previously specified magnetite/plastisol weight ratio $\mathrm{p}$. The homogeneous paste is then heated in a $180^{\circ} \mathrm{C}$ oven for about 20 minutes, allowing the solidification of plastisol. The resulting rubber-like material is flexible and molded samples can be easily cut and thinned as necessary.

Initially, two composite materials were fabricated with $\mathrm{p}=1$ and $\mathrm{p}=3$. The corresponding electromagnetic parameters were measured at $9.8 \mathrm{GHz}$ using microwave reflection techniques in a rectangular waveguide, giving the results included in Table 1. As expected, magnetic losses predominate (i.e. $\delta_{m} \gg \delta_{e}$ ). Therefore, only the design curve $\delta_{e} / \delta_{m}=0$ of Figure 2 is reproduced in Figure 3 where also the points $\left(\left(\delta_{e}+\delta_{m}\right), \varepsilon^{\prime} / \mu^{\prime}\right)$ corresponding to the absorbing materials with $\mathrm{p}=1$ and $\mathrm{p}=3$ are also represented. It is readily observed that these points lie at different sides of the design curve, meaning that the optimum magnetite concentration should have an intermediate value. Consequently, the composite was fabricated now with $\mathrm{p}=2$. The measured electromagnetic parameters are also included in Table 1 and in Figure 3 it is observed that the corresponding point $\left(\left(\delta_{e}+\delta_{m}\right), \varepsilon^{\prime} / \mu^{\prime}\right)$ is closer to the design curve, although magnetite concentration is somewhat excessive. For this reason, the magnetite/plastisol ratio was finally set at $\mathrm{p}=1.7$. The measured electromagnetic 
Table 1. Electromagnetic parameters of magnetite-plastisol composites at $9.8 \mathrm{GHz}$.

\begin{tabular}{ccccccc}
\hline $\mathrm{p}$ & $\mu^{\prime}$ & $\mu^{\prime \prime}$ & $\varepsilon^{\prime}$ & $\varepsilon^{\prime \prime}$ & $\varepsilon^{\prime} / \mu^{\prime}$ & $\delta_{e}+\delta_{m}$ \\
\hline 1.0 & 0.98 & 0.25 & 7.50 & 0.50 & 7.65 & 0.32 \\
1.7 & 0.98 & 0.40 & 11.54 & 0.61 & 11.77 & 0.44 \\
2.0 & 0.96 & 0.43 & 13.50 & 1.11 & 14.06 & 0.50 \\
3.0 & 0.90 & 0.52 & 18.40 & 1.75 & 20.44 & 0.62 \\
\hline
\end{tabular}

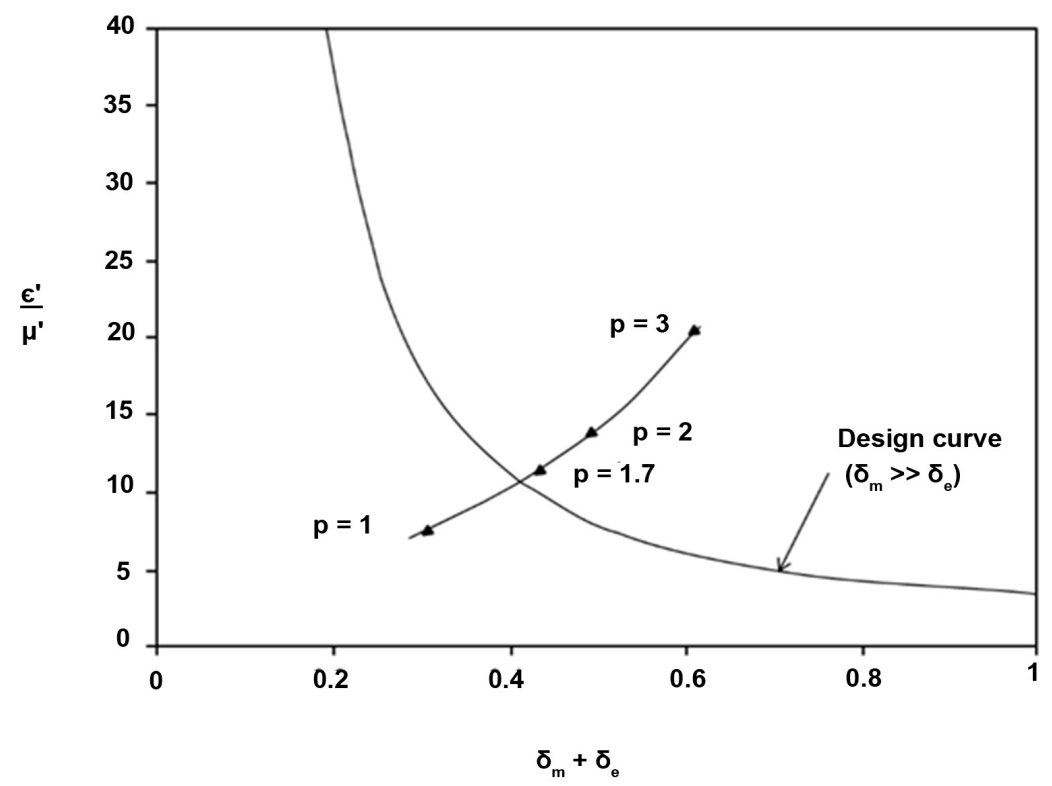

Figure 3. Representation of magnetite-plastisol composites $(p=1,1.7,2,3)$ in the design chart at $9.8 \mathrm{GHz}$.

parameters of this composite are included in Table 1 and in Figure 3 it is observed that the corresponding point is sufficiently close to the design curve making unnecessary the fabrication of composites with other intermediate magnetite/plastisol ratios.

The SLHA was fabricated with a layer of magnetite-impregnated plastisol of weight ratio $\mathrm{p}=1.7$ and thickness $\mathrm{d}=2.1 \mathrm{~mm}$ (according to the design value predicted by Equation (3)), glued to a $20 \times 20 \mathrm{~cm}$ aluminum plate. The reflection loss measured as a function of frequency in the X-band is shown in Figure 4. It is observed that the highest absorption is obtained at $9.35 \mathrm{GHz}$ instead of the design frequency (i.e. $9.8 \mathrm{GHz}$ ). This frequency shift is a consequence of not considering so far the existence of an adhesive layer between the absorber and the metallic plate. This dielectric layer, although thin, in practice has an important influence in the optimum absorption frequency. On the other hand, the influence of the finite conductivity of the aluminum plate is negligible.

In order to predict the absorption characteristics of the SLHA, the electromagnetic parameters of the magnetite-plastisol composite with $\mathrm{p}=1.7$ were measured as a function of frequency at X-band. The results for $\mu$ and $\varepsilon$ are shown in Figure 5 and Figure 6, respectively. 


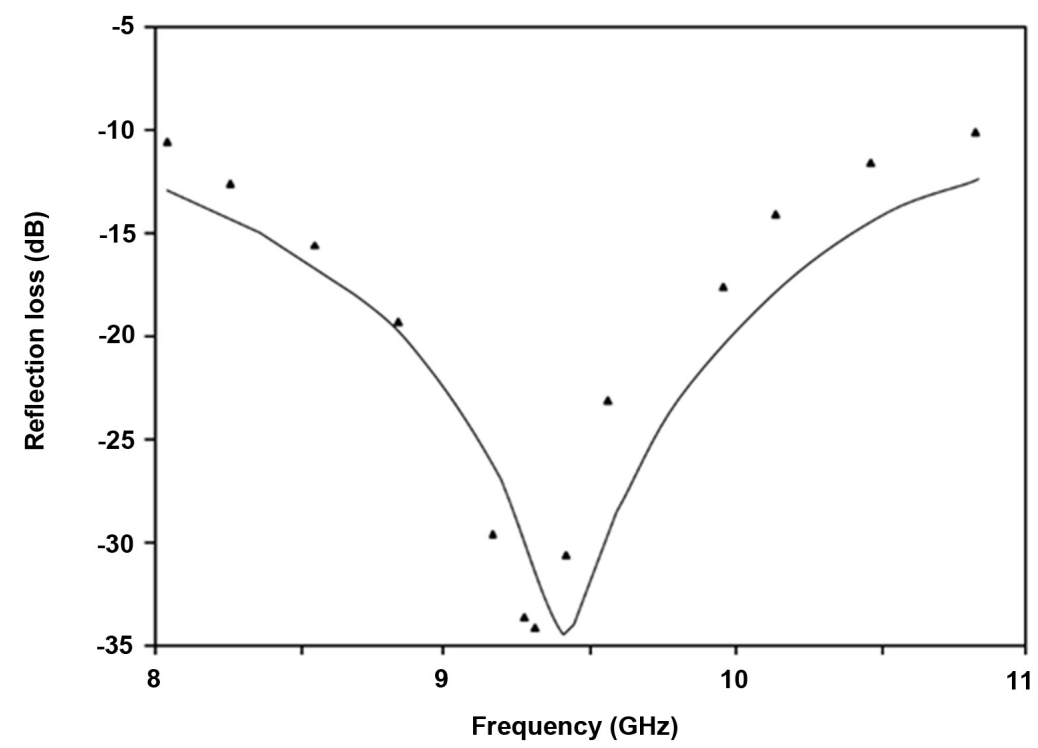

Figure 4. Absorption characteristics of the magnetite-plastisol composite ( $\mathrm{p}=$ 1.7, $\mathrm{d}=2.1 \mathrm{~mm}, \mathrm{~g}=0.1 \mathrm{~mm}) . \boldsymbol{\Delta} \boldsymbol{\Delta} \boldsymbol{\Delta}$ measured. - - calculated.

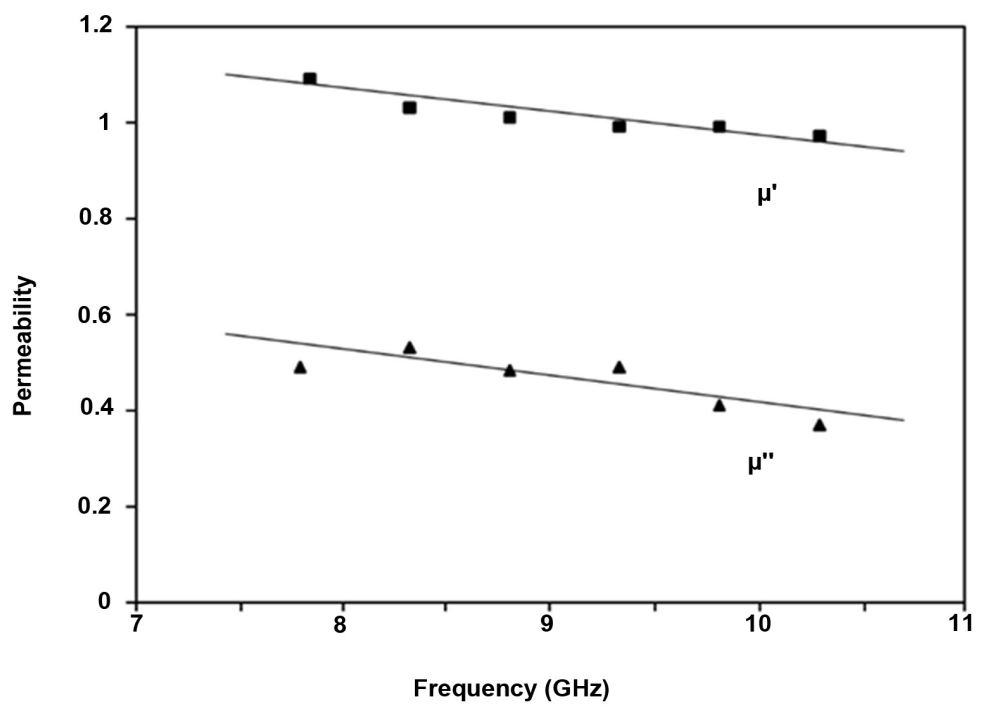

Figure 5. Permeability of the magnetite-plastisol composite $(\mathrm{p}=1.7)$ against frequency.

From the data included in Figure 5 and Figure 6, the following linear regressions are obtained:

$$
\begin{aligned}
\mu^{\prime} & =-0.045 \cdot f+1.425 \\
\mu^{\prime \prime} & =-0.055 \cdot f+0.955 \\
\varepsilon^{\prime} & =-0.004 \cdot f+11.407 \\
\varepsilon^{\prime \prime} & =-0.008 \cdot f+0.615
\end{aligned}
$$

In Equations (4)-(7) $f$ is the frequency in GHz.

Using these expressions and considering now an adhesive layer of thickness $g$ $=0.1 \mathrm{~mm}$ and relative permittivity $\varepsilon_{d}=2$, a prediction of the absorption 


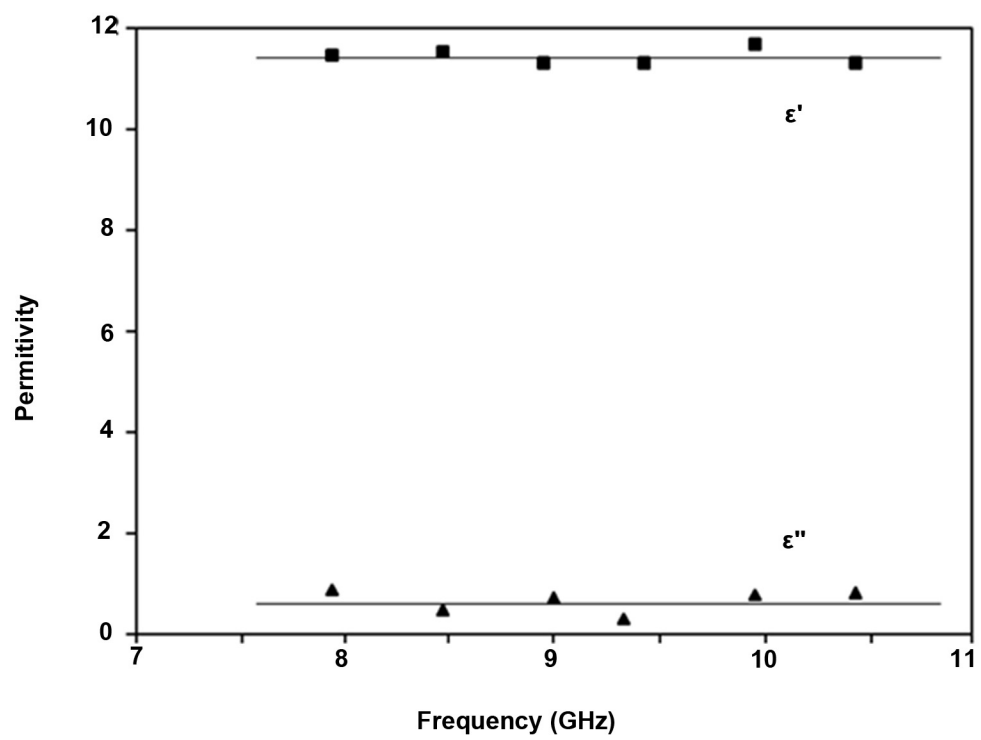

Figure 6. Permittivity of the magnetite-plastisol composite $(\mathrm{p}=1.7)$ against frequency.

characteristics of the two-layer structure was obtained (considering normal incidence of plane waves). As shown in Figure 4, good agreement between the calculated and measured values for the absorption is obtained. Consequently, in order to refine the SLHA design, the effective thickness of the absorbing material should be calculated subtracting the thickness of the adhesive layer to the value of $d$ given by Equation (3). With this procedure, maximum absorption will be obtained, approximately, at the design frequency.

In Figure 4 it is also observed that the experimental relative bandwidth of the designed SLHA is about $10 \%$ and $30 \%$ for $-20 \mathrm{~dB}$ and $-10 \mathrm{~dB}$ reflection loss, respectively.

\section{Conclusions}

A universal design method for designing single-layer electromagnetic wave absorbers under normal incidence has been presented. The method is based on a novel design chart which includes curves of constant $\delta_{e} / \delta_{m}$ ratio in a $\left(\delta_{e}+\delta_{m}, \varepsilon^{\prime} / \mu^{\prime}\right)$ plane. When representing the electromagnetic characteristics of composite absorbing materials in the design chart, it is observed that the corresponding points are very sensitive to the lossy filler concentration. Therefore, the optimum concentration at a fixed frequency is easily found after representing the measured parameters of a few trial materials with different concentrations in the design chart. Once the optimum absorbing material has been determined, the optimum thickness of the SLHA is readily calculated using a simple expression. The method has been successfully applied for designing a high performance microwave absorber using a low cost composite material made of magnetite-impregnated plastisol.

The key difference with related studies [3] and [8] is that the general design 
curves there presented show all the possible optimum parameter combinations required for perfect absorption, but do not show how far from optimum absorption is a candidate absorber, whereas the general design chart presented in this work graphically displays when a particular candidate absorber is near or will actually achieve the optimum parameter combination.

The key difference with [9] is that the particular design curves (valid only for specific values of the parameters) there presented show the optimum thickness for maximum absorption, assuming that imperfect absorption is the rule, whereas the general design chart presented in this work shows that any particular candidate absorber can achieve perfect absorption if the optimum mixture of binder and filler is provided. Once this optimum mixture is achieved a general equation gives the optimum thickness, so no curves are necessary for this purpose.

\section{Acknowledgements}

This work has been funded by the University of Chile and FONDECYT Project $\mathrm{N}^{\circ} 1940439$.

\section{Conflicts of Interest}

The authors declare no conflicts of interest regarding the publication of this paper.

\section{References}

[1] Naito, Y. and Suetake, K. (1971) Application of Ferrite to Electromagnetic Wave Absorber and Its Characteristics. IEEE Transactions on Microwave Theory and Techniques, 19, 65-72. https://doi.org/10.1109/TMTT.1971.1127446

[2] Knott, E.F. (1979) The Thickness Criterion for Single-Layer Radar Absorbents. IEEE Transactions on Antennas and Propagation, 27, 698-701. https://doi.org/10.1109/TAP.1979.1142149

[3] Fernandez, A. and Valenzuela, A. (1985) General Solution for Single Layer Electromagnetic-Wave Absorber. Electronics Letters, 21, 20-21. https://doi.org/10.1049/el:19850016

[4] Hartemann, P. and Labeyrie, M. (1987) Absorbants d'ondes électromagnétiques. Revue Technique Thomson-CSF, 19, 413-472.

[5] Musal, H.M. and Hahn, H.T. (1989) Thin-Layer Electromagnetic Absorber Design IEEE Transactions on Magnetics, 25, 3851-3853. https://doi.org/10.1109/20.42454

[6] Musal, H.M. and Smith, D.C. (1990) Universal Design Chart for Specular Absorbers. IEEE Transactions on Magnetics, 26, 1462-1464. https://doi.org/10.1109/20.104411

[7] Han, K.C., Kim, W.S. and Kim, K.Y. (1995) Practical Design Method for an Electromagnetic Wave Absorber at $9.45 \mathrm{GHz}$. IEEE Transactions on Magnetics, 31, 2285-2289. https://doi.org/10.1109/20.376231

[8] Valenzuela, A.Q. and Fernández, F.A. (1996) General Design Theory for Single-Layer Homogeneous Absorber. IEEE Transactions on Antennas and Propagation, 44, 822-826. https://doi.org/10.1109/8.510938 
[9] Giannakopoulou, T., Kontogeorgakos, A. and Kordas, G. (2003) Single-Layer Microwave Absorbers: Influence of Dielectric and Magnetic Losses on Layer Thickness. Journal of Magnetism and Magnetic Materials, 263, 173-181. https://doi.org/10.1016/S0304-8853(02)01551-2

[10] Franceschetti, G. and Silleni, S. (1964) Measurement Techniques and Experimental Data on Mixture-Type Artificial Dielectrics. Alta Frequenza, 33, 733-745.

[11] Kim, S.S., et al. (1991) Complex Permeability and Permittivity and Microwave Absorption of Ferrite-Rubber Composite in X-Band Frequencies. IEEE Transactions on Magnetics, 27, 5462-5464. https://doi.org/10.1109/20.278872

[12] Ganchev, S.I., et al. (1994) Microwave Diagnosis of Rubber Compounds. IEEE Transactions on Microwave Theory and Techniques, 42, 18-23. https://doi.org/10.1109/22.265523

[13] Kong, I., et al. (2010) Magnetic and Microwave Absorbing Properties of Magnetite-Thermoplastic Natural Rubber Nanocomposites. Journal of Magnetism and Magnetic Materials, 322, 3401-3409. https://doi.org/10.1016/j.jmmm.2010.06.036

[14] Guan, B., et al. (2017) The Electromagnetic Wave Absorbing Properties of Cement-Based Composites Using Natural Magnetite Powders as Absorber. Materials Research Express, 4, Article ID: 056103.

https://doi.org/10.1088/2053-1591/aa7025 